\title{
Single sided dipole-quadrupole magnet for the Extremely Brilliant Source storage ring at the European Synchrotron Radiation Facility
}

\author{
G. Le Bec, S. Liuzzo, F. Villar, P. Raimondi, and J. Chavanne \\ ESRF-The European Synchrotron, F-38000 Grenoble, France
}

(Received 17 January 2019; published 14 October 2019)

\begin{abstract}
Combined function magnets with dipole and quadrupole components were designed and built for the Extremely Brilliant Source. These magnets are low power consumption single sided off-axis quadrupoles. The field of the magnets was optimized for particles with a curved trajectory and within an elliptical good field region. A specific moving stretched wire magnetic measurement method was developed for measuring the magnetic length of the magnets, the radius of curvature of the poles and the field multipoles. The effect of the curvature of the magnets on the field multipoles was investigated.
\end{abstract}

DOI: 10.1103/PhysRevAccelBeams.22.102402

\section{INTRODUCTION}

The European Synchrotron Radiation Facility (ESRF), located in Grenoble, France, is a $6 \mathrm{GeV}$ light source. It is engaged in an ambitious upgrade program: the Extremely Brilliant Source (EBS). The storage ring will be completely rebuilt to reach an ultralow horizontal emittance of $135 \mathrm{pm}$ rad. The EBS storage ring is being assembled and its commissioning will start at the end of 2019. The lattice of the new ring, the so-called hybrid multibend [1,2], relies on an increased number of bending magnets and on strong focusing magnets $[3,4]$. Seven dipoles will be installed in each cell of the new storage ring: four permanent magnet dipoles with longitudinally varying field [5], and three combined function dipole-quadrupole magnets which will be described in this paper. The dipole quadrupoles of the EBS have a relatively low field $(0.39-0.57 \mathrm{~T})$ and a moderate field gradient $(31-37 \mathrm{~T} / \mathrm{m})$. The specified field and gradients implies that these magnets should be offaxis quadrupole type rather than gradient dipole type, as detailed below. The EBS dipole-quadrupole magnets have a unique feature of being asymmetric (Fig. 1): they generate a field gradient on the side of the quadrupole where the electrons travel, and almost no field on the other side. These magnets can be seen as a half quadrupole: their power consumption is almost a factor of 2 lower as compared to a standard quadrupole.

Combined function dipole quadrupoles were considered from the beginning of strong focusing synchrotrons [6]. Gradient dipoles are bending magnets with a nonuniform

Published by the American Physical Society under the terms of the Creative Commons Attribution 4.0 International license. Further distribution of this work must maintain attribution to the author(s) and the published article's title, journal citation, and DOI. gap generating a quadrupole component. They produce a strong field and a weak gradient. Such magnets were built for several accelerators including light sources [7-13]. A number of synchrotron light sources are considering upgrade schemes based on multibend lattices [14-17]. The lattices of most of these light sources include combined function magnets with strong quadrupole components, similar to the EBS ones.

Determining which type of magnet, among gradient dipoles or quadrupoles, is the best for given specifications is the starting point of the design. Let us first consider a gradient dipole magnet. The field of a nonsaturated dipole is approximately $B_{0}=\mu_{0} N I / g$, where $N I$ is the number of turns times the current and $g$ is the magnet gap. The field is affected by a variation of the gap as $\Delta B / B=-\Delta g / g$ and a gap variation translate to a field gradient $G$ as

$$
G=-\frac{B}{g} \frac{\Delta g}{\Delta x} .
$$

If the pole width is equal to twice the gap, the upper pole touches the lower pole if $\Delta g / \Delta x=1$. The corresponding gradient is

$$
G=\frac{B}{g} .
$$

This gives an overestimated upper bound for the gradient. Considering the field and gap of the DQ2 magnet of the EBS, i.e., $B \approx 0.4 \mathrm{~T}$ and $g \approx 25 \mathrm{~mm}$, the corresponding gradient is $16 \mathrm{~T} / \mathrm{m}$, far from the $31 \mathrm{~T} / \mathrm{m}$ DQ2 specification. Equation (2) shows that gradient dipoles are not suitable for high gradient, low field magnets. Quadrupoles with transverse offsets are much better for that purpose. Figure 2 shows fields and gradients for different types of combined function magnets. It clearly appears that high gradient low field magnets are quadrupoles and high field low gradient magnets are gradient dipoles. 


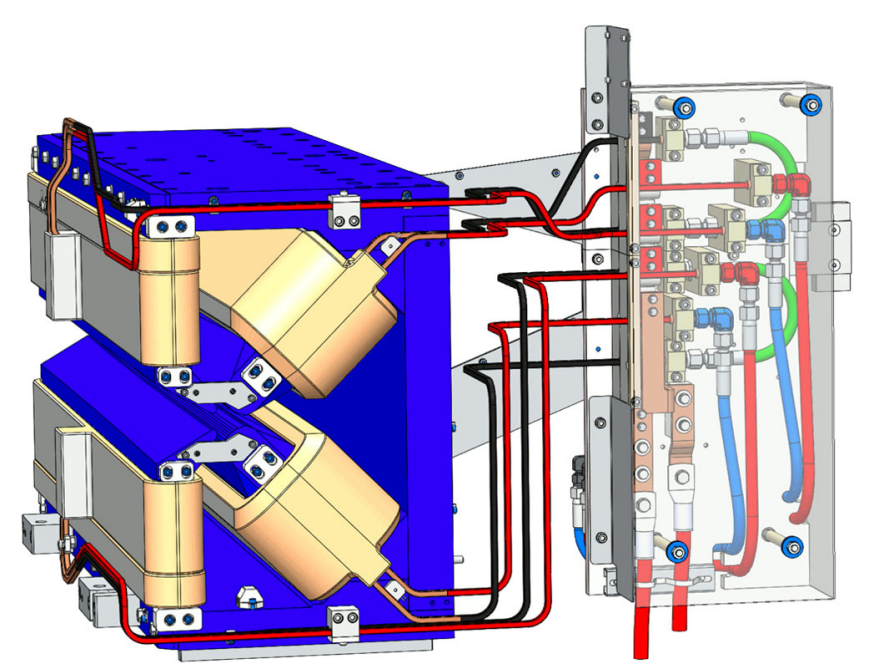

FIG. 1. Design view of the DQ1 magnet. This magnet is a single sided quadrupole: the field on the outer part of the magnet is almost zero. It can be seen as a half quadrupole magnet and it consumes less power than a standard symmetric quadrupole. The coils are shared between the main poles and the small auxiliary coils in order to increase the power efficiency of the magnet. Additional low current coils were added to trim the field and the gradient independently.

The design of the EBS dipole quadrupoles will be detailed in the next section. The concept of a single sided quadrupole will be introduced and then the magnetic models and the optimization process will be described.

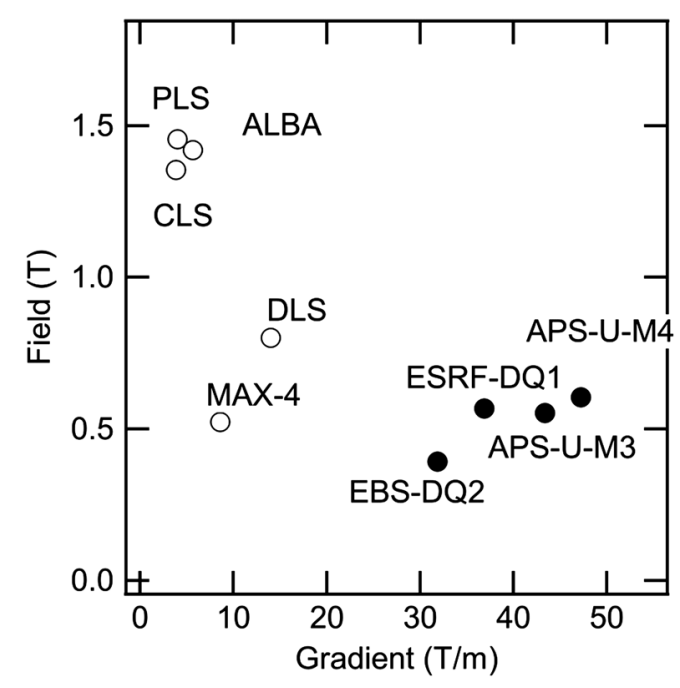

FIG. 2. Fields and gradients of combined dipole quadrupoles installed in light sources. The disks indicate quadrupole type magnets and the circles indicate gradient dipole types. The Canadian Light Source (CLS), the Spanish light source ALBA and the Pohang Light Source (PLS) are third generation light sources, while MAX-4, the EBS and the Advanced Photon Source are next generation light sources. The combined function magnets for the EBS and the APS upgrade are quadrupole types while the magnets of previous light sources are gradient dipoles.
The field and gradient trimming method will be presented, as well as the sensitivity of the field to mechanical errors.

The EBS dipole quadrupoles were built and are being assembled in the storage ring. Measurement results for some of these magnets are shown in Sec. III. Hall probe field measurements as well as stretched wire integrated field measurements were performed. A specific stretched wire method was developed for the dipole quadrupoles; this method is detailed in the same section.

The curvature of the magnet complicates the analysis of the magnetic field. In particular, the series expansions commonly used to describe the field integrals of straight magnets, or the 2D fields of long magnets, is inaccurate for curved magnets. This topic is discussed in Sec. IV.

\section{DIPOLE-QUADRUPOLE DESIGN}

\section{A. The "DQ" layout}

The main specifications of the EBS dipole quadrupoles (DQs) are given in Table I. Given the magnet apertures, the gradients are strong enough to exclude a gradient dipole design. As the deflection of the electron's trajectory is far from being negligible, we decided to build magnets with curved pole surfaces. The iron length of the magnet was fixed from the beginning, the distance between the coils of adjacent magnets being set to approximately one centimeter.

The cross section of the EBS dipole-quadrupole magnets is sketched in Fig. 3. It has been designed as a gradient dipole magnet with a small additional pole for improving the homogeneity of the gradients. It could also be seen as a quadrupole septum [18], with its magnetic mirror plate deformed and opened in the horizontal symmetry plane.

It would have been possible to build the dipole quadrupoles as curved, offset quadrupoles. This solution was chosen by others, e.g., the APS-U team [14]. Offset quadrupoles have some advantages, mainly their simpler

TABLE I. Lattice specifications for the DQ1 and DQ2 magnets of the EBS. The central field and gradient specified here are initial values obtained from the integrated strengths and the iron length.

\begin{tabular}{lccc}
\hline \hline & DQ1 & DQ2 & \\
\hline Iron length & 1028 & 800 & $\mathrm{~mm}$ \\
Integrated field & 584.4 & 314.1 & $\mathrm{~T} \mathrm{~mm}$ \\
Integrated gradient & 38.43 & 25.25 & $\mathrm{~T}$ \\
Central field & 0.5683 & 0.3926 & $\mathrm{~T}$ \\
Central gradient & 37.38 & 31.56 & $\mathrm{~T} / \mathrm{m}$ \\
Angle @ 6 GeV & 29.2 & 15.7 & $\mathrm{mrad}$ \\
Radius of curvature & 35207 & 51955 & $\mathrm{~mm}$ \\
Sagitta of the poles & 3.88 & 1.60 & $\mathrm{~mm}$ \\
Bore radius & 12.5 & 12.5 & $\mathrm{~mm}$ \\
GFR radius & $7 \times 5$ & $7 \times 5$ & $\mathrm{~mm} \times \mathrm{mm}$ \\
$\Delta G / G$ @ 7 mm & $10^{-2}$ & $10^{-2}$ & \\
Number of magnets & 64 & 32 & \\
\hline \hline
\end{tabular}




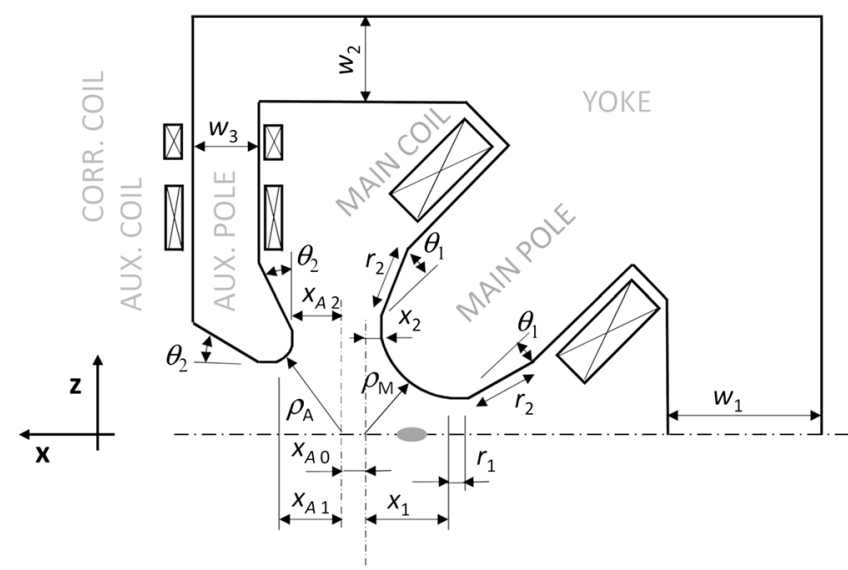

FIG. 3. Sketch of the dipole-quadrupole cross section, showing the main parameters of the magnet. The main coil and the auxiliary coils are serially connected. An additional correction coil is installed close to the auxiliary pole. The gray ellipse below the main pole indicates the approximate position of the electron beam.

magnetic design and optimization and their better behavior in terms of vibrations and mechanical deflection. The advantages of the DQs presented here are their low power and the side access which eases the measurements and the integration.

\section{B. Magnetic field computations}

The magnetic fields of the DQ magnets were computed with Radia, a 3D magnetostatic code developed at the ESRF $[19,20]$. The optimization method employed for the pole shaping has been presented in other papers [4,21] and will not be presented in detail here. Any reader interested in field computations and pole optimization may refer to [22].

The main geometric parameters of the DQs are shown in Fig. 3. At the beginning of the optimization process, the poles had a hyperbolic shape following the equation $x_{M}=$ $\rho_{M}^{2} / 2 z_{M}$ for the main pole, $\left(x_{M}, z_{M}\right)$ being the position of a vertex point of the main pole profile, and $x_{A}-x_{A 0}=$ $\rho_{A}{ }^{2} / 2 z_{A}$ for the auxiliary pole described by the vertex points $\left(x_{A}, z_{A}\right)$. Two perpendicular reference planes were defined on each pole. The width of these planes is $r_{1}=3 \mathrm{~mm}$. The outer parts of the yoke are straight, but the poles are curved.

First, the parameters $\theta_{1}, \theta_{2}, r_{2}, \rho_{A}, w_{1}, w_{2}, w_{3}, x_{1}, x_{A 0}$, $x_{A 1}$ and $x_{A 2}$ were determined manually in order to get the field and the gradient close to the specifications, regardless of the homogeneity.

An optimization criterion was defined using a combination of the exact values of the field and gradient, the gradient homogeneity on an elliptical $14 \times 10 \mathrm{~mm}^{2}$ good field region (GFR), and minimal values for the vertical apertures between the magnet poles.
The gradient homogeneity criterion was set with elliptic multipoles (see Refs. [23,24] and the Appendix). A vector multipole error was defined as

$$
\varepsilon=\mathbf{E}-\mathbf{E}_{0}=\mathbf{M}_{\mathbf{E}}^{+}\left(\mathbf{B}-\mathbf{M}_{\mathbf{C}} \mathbf{C}_{\mathbf{0}}\right),
$$

where $\mathbf{M}_{\mathbf{E}}$ and $\mathbf{M}_{\mathbf{C}}$ are defined in the Appendix, $\mathbf{M}_{\mathbf{E}}{ }^{+}$is a pseudoinverse of $\mathbf{M}_{\mathbf{E}}$ and $\mathbf{C}_{\mathbf{0}}=\left(B, G \rho_{0}, 0, \ldots, 0\right)^{T}$ contains the field and gradient specifications. The multipole expansions used here are valid for 2D fields. They are correct for the integral of the field along a straight line, and far from the edges of a long, straight magnet. But the poles of the DQs follow the reference trajectory of the electrons: they are curved. At this stage of the design, the curvature of the poles was taken into account using an empirical method. Integrals of the vertical field components were integrated according to the path shown in Fig. 4. These field integrals were computed at sixteen points $\left(x_{i}, z_{i}\right)$ located on a half ellipse, where $x_{i}$ and $z_{i}$ are the horizontal and vertical offsets of the path $i$. The error vector defined in Eq. (3) was computed at each iteration using these field integrals.

There is no reason for the line-arc field integrals described here to be expandable in Taylor series. The multipole errors are expected to be in the order of $\rho_{0} / R_{0}, \rho_{0}$ being the reference radius and $R_{0}$ the bending radius [24], and this number is well below the homogeneity tolerance. The effect of the magnet's curvature will be discussed in more detail in Sec. IV.

The homogeneity of the gradient, for the optimized DQ1 geometry, is shown in Fig. 5. The field was integrated along paths parallel to the reference electron trajectories [Fig. 4(b)], then differentiated to get the relative integrated gradient error $\Delta G / G$. The inhomogeneities appear to be 1 order of magnitude below the specifications within the good field region. The elliptical shape of the good field region is visible on the plot. The first six circular multipole coefficients are given in Table II.

Figure 6 shows the vertical field of the magnet in its central plane. This field is similar to the field of a quadrupole magnet for $x<15 \mathrm{~mm}$. At higher values of $x$ (i.e., on the outer part of the ring) the field is close to zero.

The field and gradient along the reference axis are plotted in Fig. 7. It shows that the magnetic lengths for the dipole and the quadrupole terms are not the same. Using the integrated strength divided by the central strength as an approximation of the magnetic length, one gets the dipole length $L_{1} \approx 1054.7 \mathrm{~mm}$ and the quadrupole length $L_{2} \approx 1044.0 \mathrm{~mm}$, the iron length being $1028 \mathrm{~mm}$. Indeed, a quadrupole field decreases faster than a dipole field. The ratio between the two magnetic lengths is driven by the magnet bore radius and by the iron length.

Two additional air-cooled coils, named correction coils, were added on the bottom and top auxiliary poles. The purpose of the correction coils is to add a knob to tune the 


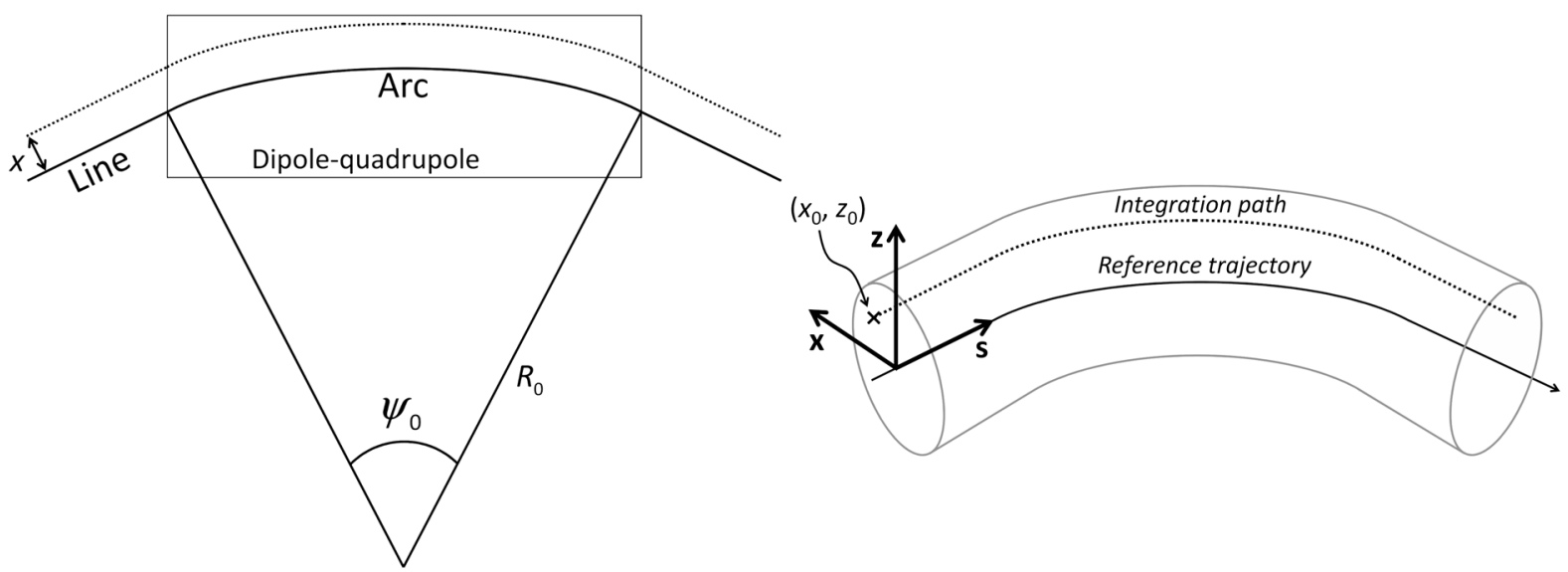

(a)

(b)

FIG. 4. (a) Field integration path used for the shape optimization. The path was built with a line, an arc corresponding to the magnet deflection angle $\psi_{0}$ and another line. The bending radius is $R_{0}$. The solid line is the reference trajectory and the dotted line is a path with horizontal offset $\mathrm{x}$ and vertical offset $\mathrm{z}$. These paths have the advantage of being independent of the magnet design: this facilitates the optimization, as it is stable from one iteration to the next. (b) Coordinate system and integration path used for field and integrated field computations, after the optimization process. The path is parallel to the reference trajectory computed by a tracking code. The $\mathrm{s}$ axis is parallel to the trajectory, the $\mathrm{x}$ axis is horizontal and perpendicular to the reference trajectory, and the $\mathrm{z}$ axis is perpendicular to $\mathrm{s}$ and $\mathrm{x}$. A given integration path is displaced by $\left(x_{0}, z_{0}\right)$ with respect to the reference trajectory.

field and the gradient of the dipole quadrupole independently. Maps of the integrated field, gradient and sextupole are shown in Fig. 8.

The integrated fields and gradients have an almost linear dependence on the main and corrector current. Their strengths can be approximated with a matrix whose coefficients are obtained by fitting a plane to the field and gradient maps. Simulations have shown that the relative errors between this simple model and the nonlinear

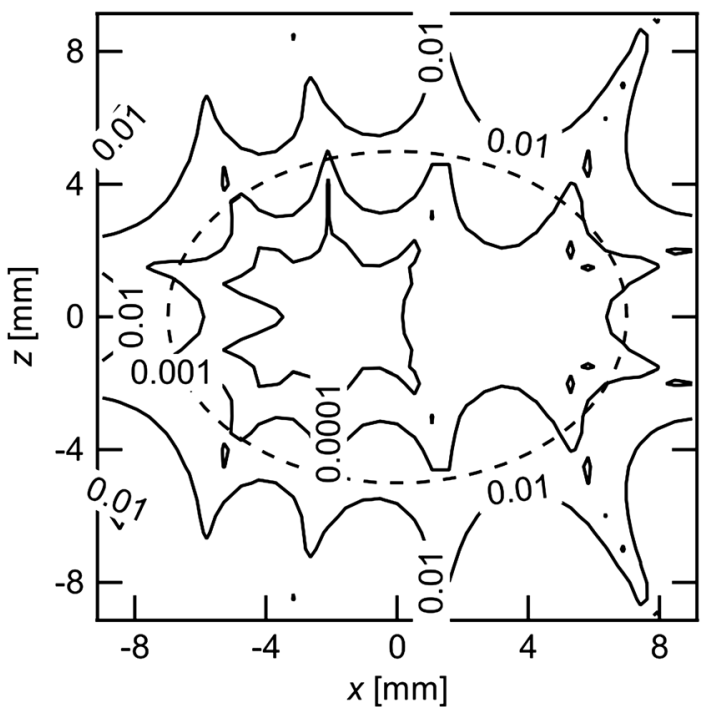

FIG. 5. Gradient inhomogeneities $\Delta G / G$ integrated along a path parallel to the reference trajectory [Fig. 4(b)], for the DQ1 magnet. In the horizontal symmetry plane, the gradient is homogeneous to within $0.1 \%$ in a $\pm 6.1 \mathrm{~mm}$ range. The dashed curve indicates the $14 \times 10 \mathrm{~mm}$ GFR. magnetic simulations is below $10^{-3}$ for the current ranges shown in Fig. 8. A better approximation would have been obtained by fitting a higher order surface to the maps, e.g., a paraboloid.

Changing the field and gradient introduces a sextupole component, as shown in Fig. 8. The sextupole strength varies almost linearly with the corrector current and is relatively independent of the main coil current. At seven millimeters from the center of the magnet and at the maximum corrector current, the ratio of the integrated sextupole to the dipole reaches $\beta_{3} / \beta_{1} \approx 3 \times 10^{-3}$ which is not negligible. It should be noted that adding a correction coil in an offset quadrupole magnet would have introduced a similar sextupole component.

TABLE II. First normal circular multipole coefficients for the DQ1 magnet, normalized to the dipole coefficient and expressed at $7 \mathrm{~mm}$ (the $\beta_{n}$ denotes "pseudomultipole coefficients" integrated along a curved path. These multipoles are introduced in Sec. III. The standard deviations $\sigma_{n}$ of the multipole errors $\beta_{n}$ were computed for $a \pm 0.05 \mathrm{~mm}$ tolerance equally shared between assembly and machining errors. One hundred sample dipole-quadrupole magnets were simulated. The field was integrated along paths parallel to the reference trajectory.

\begin{tabular}{lcc}
\hline \hline$n$ & $\beta_{n}$ & $\sigma_{n}$ \\
\hline 1 & 10000 & $(41)$ \\
2 & 4550.8 & $(19.7)$ \\
3 & 2.5 & 7.7 \\
4 & -3.7 & 3.9 \\
5 & -2.7 & 2.1 \\
6 & 9.0 & 1.6 \\
\hline \hline
\end{tabular}




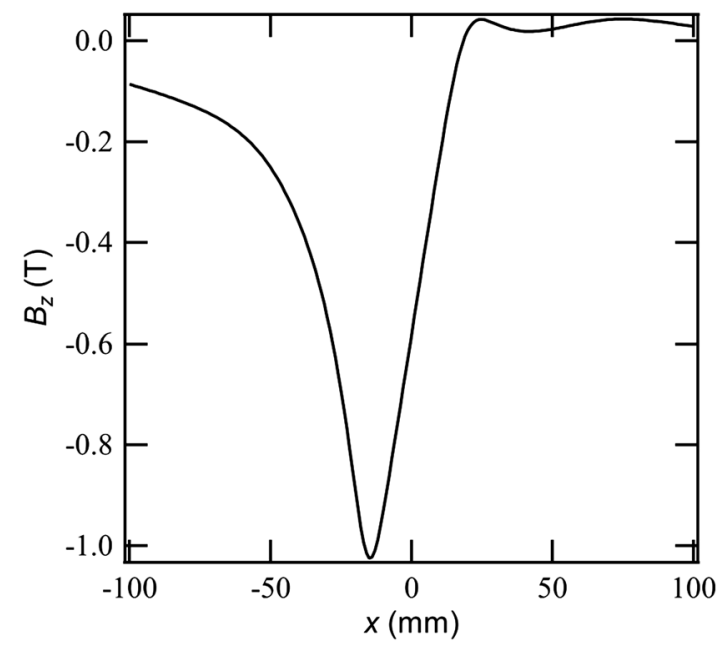

FIG. 6. Vertical field of the DQ1 dipole-quadrupole magnet in the longitudinal symmetry plane. The nominal position of the beam is $x=0$.

Random pole displacements and random pole shape errors were introduced in the magnetic model in order to simulate assembly and machining errors. Table II shows the sensitivity for the DQ1, assuming a $\pm 0.050 \mathrm{~mm}$ mechanical tolerance. The relatively high values for these standard deviations are driven by the small bore radius of a magnet, as we discussed in Ref. [4]. The dipole term has the largest standard deviation. It translates to a center position uncertainty of $64 \mu \mathrm{m}$. The standard deviation of the quadrupole term gives the gradient error $\Delta G_{0} / G_{0}=4.3 \times 10^{-3}$ for the DQ1. In practice, these two errors depend on the characterization of the magnet: the error on the position of the magnet center is measured and enforced to zero by the fiducialization, and the current is set in order to reach the nominal gradient. The first significant multipole error is the sextupole, with a $\sigma_{3}=7.7 \times 10^{-4}$ standard deviation

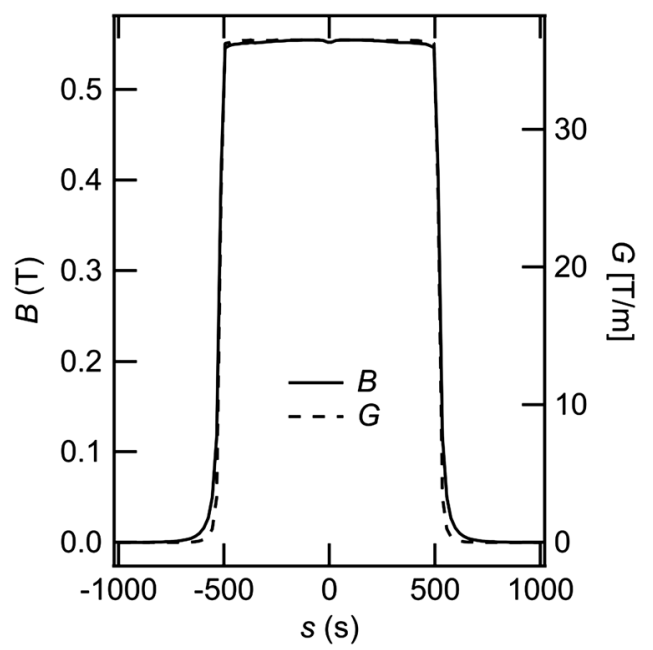

FIG. 7. Vertical field and gradient along the reference trajectory. Notice the longer magnetic length for the dipole field.
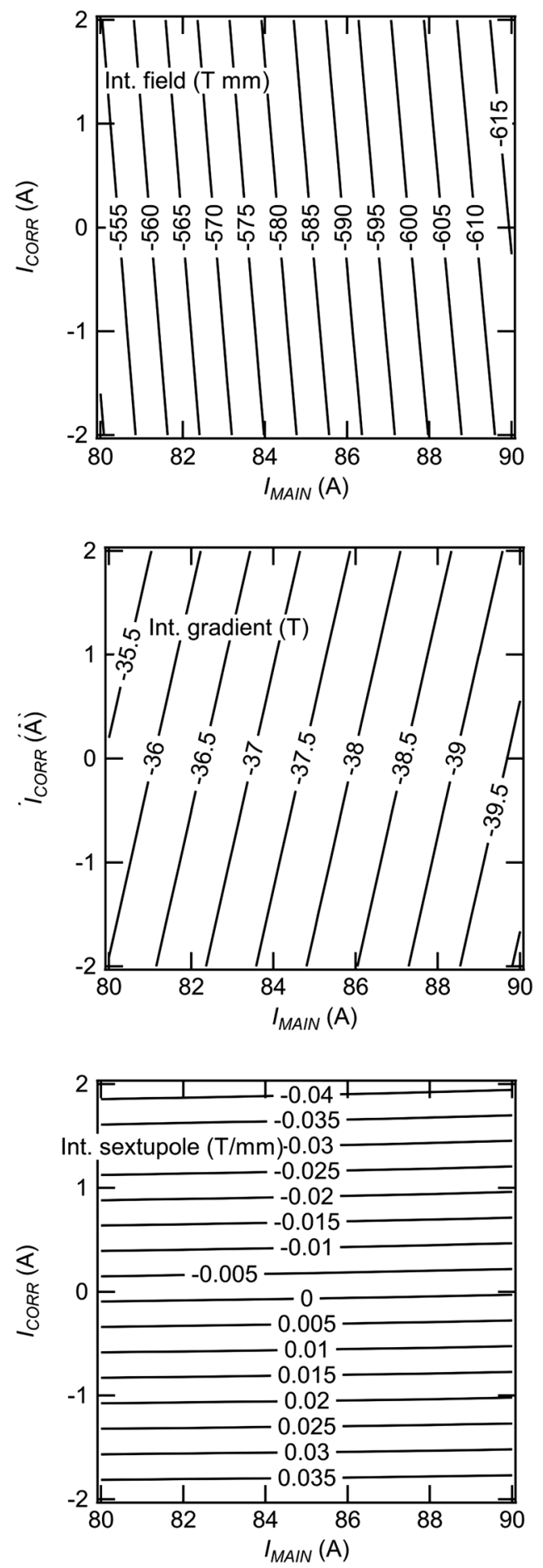

FIG. 8. Field and gradient trimming using correction coils: integrated field (top), integrated gradient (middle) and integrated sextupole strength defined as $\beta_{3} / \rho_{0}{ }^{2}$ (bottom). The strengths were integrated along paths parallel to the reference trajectory. At $7 \mathrm{~mm}$ from the center of the magnet and at the maximum corrector current $(2 \mathrm{~A}), \beta_{3} / \beta_{1} \approx 0.003$.

(normalized to the dipole). Beam dynamics simulations have shown that, with these levels of higher order multipole errors, the impact on the lifetime and dynamic 
aperture is small. In these conditions, assuming $0.05 \mathrm{~mm}$ rms alignment errors and taking into account random and systematic errors on all magnet families, the Touschek lifetime is $22.6 \pm 1.1$ hours, the dynamic aperture is $8.5 \pm$ 0.4 millimeters and the injection efficiency is $91.6 \pm 3.2 \%$. These values are acceptable for the EBS.

\section{Engineering design}

The reduction of the electrical consumption of the accelerator was one of the goals of the ESRF upgrade. As the magnets are major contributors to the electrical bill, a substantial effort was made to decrease their consumption.

The power efficiency of the dipole quadrupoles is improved by a factor of 2 compared to a quadrupole with similar gradient and aperture because the DQs are single sided magnets. The power consumption can be reduced further by decreasing the current density $\left(3 \mathrm{~A} / \mathrm{mm}^{2}\right.$ for the DQ1). It results in bigger coils, which means more copper and less compact magnets. The DQ1 main coil has 65 turns and its auxiliary coil has 12 turns. The main coils and auxiliary coils are connected in a series and are water cooled. The magnet power is $1.4 \mathrm{~kW}$ at nominal current of $85 \mathrm{~A}$. The correction coils are air cooled and their power consumption is negligible.

Figure 1 shows a design view of the DQ1 magnet. The yoke is made with seven AISI 1006 low carbon plates. The repeatability of the yoke and pole assembly is ensured by the design of the mating surfaces (Fig. 9). Most of the magnet parts are straight, but the pole surfaces are curved, following the reference particle trajectory.

The vertical magnetic force applied by the two lower poles on the two upper poles is $22 \mathrm{kN}$. This force creates a closure of the gap by $100 \mu \mathrm{m}$. This gap reduction is not a big issue, since the dipole quadrupoles will be powered in a limited range around the nominal current: the deformation can be compensated by machining the backleg of the yoke $100 \mu \mathrm{m}$ higher than its nominal dimension height.

Due to the absence of yoke on one side, the DQs are less rigid than standard quadrupoles. It would have been possible to improve the rigidity by installing nonmagnetic strengthening parts on the open side of the magnet, at the cost of a reduction of the side access to the magnet gap. Another solution would be to increase the thickness of the yoke. The first vibration mode of the DQ1 was found at $107 \mathrm{~Hz}$, which was considered to be high enough.

\section{MAGNETIC MEASUREMENTS}

\section{A. Stretched wire measurements of the integrated field}

Flux meters are often used to map the integrated field of accelerator magnets. Rotating coils have been used for decades and are considered as the reference tools for multipole magnets [25]. Stretched-wire systems, initially

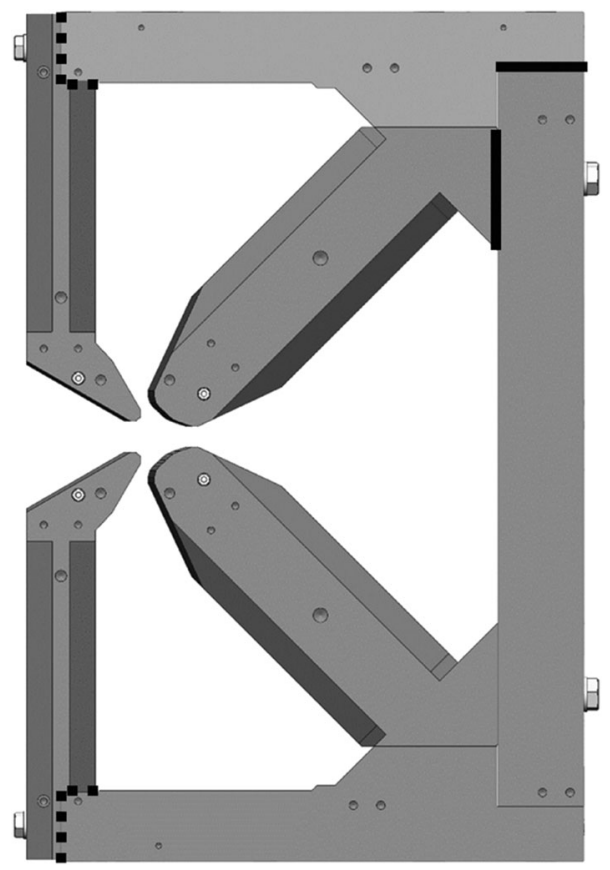

FIG. 9. Yoke of the DQ1 magnet. The yoke is an assembly of seven parts machined in low carbon plates. The bold solid lines indicate the mating surfaces used for positioning the top of the magnet. The bold dotted lines indicate the mating surface used for positioning the auxiliary poles which must be removed for inserting the coils.

designed for alignment [26,27] and insertion devices measurements [28], can now compete with rotating coils for multipole field mapping [29].

Unfortunately, none of these solutions are satisfactory for measuring curved magnets. In a dipole magnet, the field integral along a straight line has no significance in terms of beam dynamics - even if it may be used for field quality control, by comparison with simulations. Curved coils have been built by other groups [30]. Such coils are well adapted to measure the strength of a dipole field. In the case a coil is linearly displaced within a field gradient, the accuracy of the measurement is limited [29]. Another possibility is to measure field multipoles at different locations with a rotating coil shorter than the magnet and moved along the reference axis of the magnet [31].

We developed another method which combines a simplified model of the magnetic field and stretched-wire measurements. In the first instance, let us assume that the magnetic field is a pure dipole-quadrupole field within a straight magnetic length $L_{S 1}$ for the dipole and $L_{S 2}$ for the quadrupole and is zero elsewhere (Fig. 10), and that the bending radius of the poles is $R_{0}$ (Fig. 4). With this simplified model, the field along the reference trajectory has a rectangular shape. Using the notations introduced in the figures, the vertical component of the field can be written as 

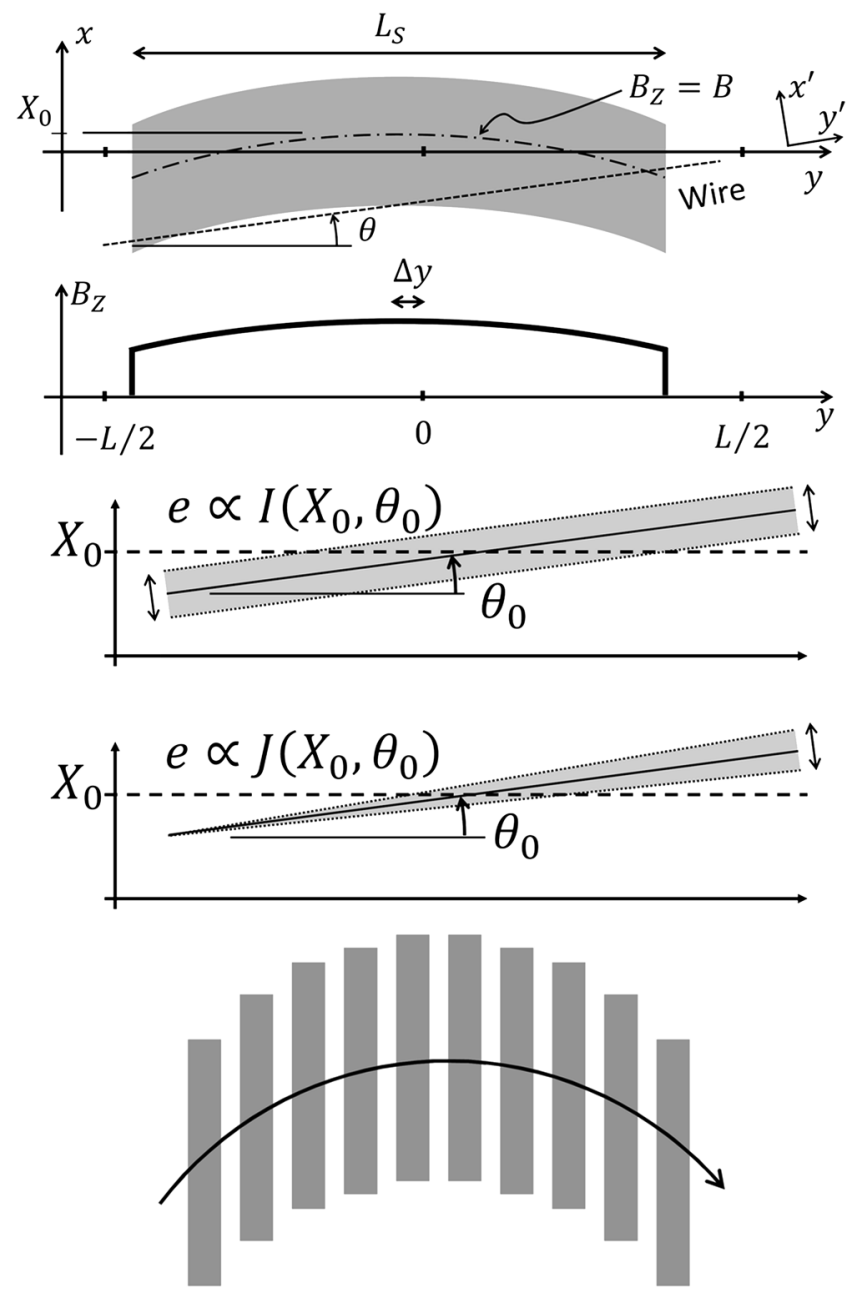

FIG. 10. Top: Simplified field model used for the measurement of the dipole-quadrupole magnets. The field is assumed to be nonzero over a length $L_{S}$ and the radius of curvature of the magnet poles is $R_{0}$. The magnet is measured using a stretched wire of length $L$. The longitudinal distance between the magnet and the wire center is $\Delta y$. The dashed line represents the wire. The magnet frame is $(x, y)$ and the wire frame is $\left(x^{\prime}, y^{\prime}\right)$. Middle: If the two extremities of the wire are moved in the same direction, the induced voltage e is proportional to the integral of the field $I\left(X_{0}, \theta_{0}\right)$. If only one extremity of the wire is moved, the induced voltage varies with the second integral of the field $J\left(X_{0}, \theta_{0}\right)$. Bottom: In the "pseudocurvilinear multipole" approximation developed in this paper, the field of the dipole-quadrupole magnet is modeled as a succession of parallel slices. In each slice, the field is approximated by an analytic function, i.e., a Taylor series. The multipole coefficients are assumed to be the same for all slices.

$$
B_{Z}(x, y) \approx B+G\left(x-X_{0}+\theta y+\frac{(y-\Delta y)^{2}}{2 R_{0}}\right),
$$

where the quadratic term approximates the curvature of the magnet with a parabola and $\theta$ is the angle between the magnet axis and the wire axis. The integral along the wire can be computed analytically. Assuming $\theta$ small and neglecting the terms in $\theta^{2}$ when integrating along the magnet axis instead of the wire axis [i.e., $d y^{\prime}=$ $\left.\cos \theta d y \approx\left(1-\theta^{2} / 2\right) d y \approx d y\right]$, the integral of the field is

$$
\begin{aligned}
& I_{Z}(x, \theta) \\
& \quad=\int_{-L / 2}^{L / 2} B_{Z} d y^{\prime} \approx \int_{-L / 2}^{L / 2} B_{Z} d y \\
& \quad \approx B L_{S 1}+G \int_{-L_{S 2} / 2+\Delta y}^{L_{S 2} / 2+\Delta y}\left(x-X_{0}+\theta y+\frac{(y-\Delta y)^{2}}{2 R_{0}}\right) d y \\
& \quad \approx B L_{S 1}+G L_{S 2}\left(\frac{L_{S 2}^{2}}{24 R_{0}}+x-X_{0}+\theta \Delta y\right)
\end{aligned}
$$

A second integration gives

$$
\begin{aligned}
J_{Z}(x, \theta) \approx & \int_{-L / 2}^{L / 2} \int_{-L / 2}^{v} B_{Z} d y d v \\
\approx & B L_{S 1}(L / 2-\Delta y)+G L_{S 2}\left[\frac{L}{2}\left(x-X_{0}+\frac{L_{S 2}^{2}}{24 R_{0}}\right)\right. \\
& -\frac{\Delta y}{2}\left(\frac{L_{S 2}^{2}}{12 R_{0}}-\theta L+2 x-2 X_{0}\right) \\
& \left.-\theta\left(\frac{L_{S 2}^{2}}{12}+\Delta y^{2}\right)\right]
\end{aligned}
$$

Moving the two extremities of the wire in the same direction gives the $I_{X, Z}$ integrals, while moving only one extremity of the wire gives the second integrals $J_{X, Z}$ [28]. The field integral and second field integral measurement methods are sketched in Fig. 10.

Let us first measure the field integral at two wire positions $I_{Z 01}=I_{Z}\left(\Delta X, \theta_{0}\right)$ and $I_{Z 02}=I_{Z}\left(-\Delta X, \theta_{0}\right)$, the axis of the magnet being determined by the unknown position $X_{0}$ and angle $\theta_{0}$. The difference of these two integrals simplifies to $I_{Z 01}-I_{Z 02}=2 G L_{S 2} \Delta x$, so the integrated gradient is

$$
G L_{S 2}=\frac{I_{Z 01}-I_{Z 02}}{2 \Delta x}
$$

The transverse position and angle and the above field integrals satisfy

$X_{0}=\frac{1}{G L_{S 2}}\left(B L_{S 1}-\frac{I_{Z 01}+I_{Z 02}}{2}\right)+\frac{L_{S 2}^{2}}{24 R_{0}}+\theta_{0} \Delta y$.

The sagitta of the trajectory is $\mathcal{S} \approx L_{S}{ }^{2} / 8 R_{0}$ and in the case $\left(\theta_{0}, \Delta y\right)=\mathbf{0}$ this equation simplifies to 


$$
X_{0}=\frac{1}{G L_{S 2}}\left(B L_{S 1}-\frac{I_{Z 01}+I_{Z 02}}{2}\right)+\frac{\mathcal{S}}{3} .
$$

This result was first demonstrated by Jain [32]. Then, let us measure the field integrals at $X_{0}$ and $\theta_{0} \pm \Delta \theta$ : $I_{Z 11}=I_{Z}\left(X_{0}, \theta_{0}+\Delta \theta\right), J_{Z 11}=J_{Z}\left(X_{0}, \theta_{0}+\Delta \theta\right), I_{Z 12}=$ $I_{Z}\left(X_{0}, \theta_{0}-\Delta \theta\right)$ and $J_{Z 12}=J_{Z}\left(X_{0}, \theta_{0}-\Delta \theta\right)$. Combining the first field integrals gives the longitudinal position of the magnet,

$$
\Delta y=\frac{I_{Z 11}-I_{Z 12}}{2 G L_{S 2} \Delta \theta},
$$

while the straight magnetic length is deduced from the differences of the second field integral:

$L_{S 2}=\sqrt{\frac{6}{G L_{S 2} \Delta \theta}\left(J_{Z 12}-J_{Z 11}\right)+6 \Delta y(L-2 \Delta y)}$.

The length of the curved reference path can be deduced using the following series expansion:

$$
L_{C 2} \approx L_{S 2}\left(1+\frac{L_{S 2}^{2}}{24 R_{0}^{2}}\right) \text {. }
$$

The wire yaw angle is computed from a combination of the first and second field integrals:

$\theta_{0}=\frac{6 L}{G L_{S 2}{ }^{3}}\left[\left(I_{Z 11}+I_{Z 12}\right)(L / 2-\Delta y)-\left(J_{Z 11}+J_{Z 12}\right)\right]$.

The position $X_{0}$ is then obtained by inserting in Eq. (8) the values $\theta_{0}$ obtained from Eq. (13) and $\Delta y$ obtained from Eq. (10).

All of the above parameters were obtained with an a priori value for $R_{0}$. Let us now compare the second integrals $J_{Z 11}$ and $J_{Z 12}$, obtained on a magnet whose curvature radius is $R_{0}+\Delta R_{0}$, to the a priori values $J_{Z 110}$ and $J_{Z 120}$ computed assuming a radius $R_{0}$. From a series expansion of Eq. (6), we get

$\Delta R_{0} \approx \frac{24 R_{0}^{2}}{G L_{S 2}{ }^{3}(L-2 \Delta y)}\left(J_{Z 110}+J_{Z 120}-J_{Z 11}-J_{Z 12}\right)$.

The radius of curvature can thus be obtained from the difference between the measured second field integrals and their value assuming $R_{0}$.

One should note that the above method can be used for quadrupole alignment, by setting $B=0$ and $R_{0} \rightarrow \infty$. The vertical position and the pitch angle of the magnetic axis are obtained in this particular case.
We have shown that a few first and second field integral measurements fully determine the magnet alignment, including its longitudinal position and the pitch and yaw angles. The same measurements give most of the parameters of the model: the field gradient, the quadrupole magnetic length and the radius of curvature of the poles. The dipole magnetic length, not determined here, can be taken as being equal to the quadrupole length as a first approximation.

Let us now assume that the wire is aligned on the magnet axis. In the case where the magnetic field is not a pure dipole quadrupole but contains higher order multipoles, the field integral can be approximated by

$$
\begin{aligned}
I_{Z}+i I_{X} & =\int\left(B_{Z}+i B_{x}\right) d l=\sum_{n>0}\left(b_{n}+i a_{n}\right)\left(\frac{u}{r_{0}}\right)^{n-1} \\
& \approx \sum_{n>0} \frac{\beta_{n}+i \alpha_{n}}{L_{S} r_{0}{ }^{n-1}} \int_{-L_{S} / 2}^{L_{S} / 2}\left(u+\frac{y^{2}}{2 R_{0}}\right)^{n-1} d y
\end{aligned}
$$

where $u=x+i z$ is the complex variable, $a_{n}$ and $b_{n}$ are the multipole coefficients of the integrated field (see the Appendix) and $\beta_{n}$ and $\alpha_{n}$ are "pseudocurvilinear multipole" coefficients integrated along the curved trajectory. The arc is approximated by the parabola $x=y^{2} /\left(2 R_{0}\right)$.

We neglect here the fringe field terms. The effect of these terms will be discussed in Sec. IV.

Applying the binomial formula to Eq. (15) and integrating leads to

$$
\begin{aligned}
I_{Z}+i I_{X} \approx & -\sum_{n>0} \sum_{k=0}^{n-1}\left(\beta_{n}+i \alpha_{n}\right)\left(\begin{array}{c}
n-1 \\
k
\end{array}\right) \\
& \times\left(\frac{L_{S}^{2}}{8 R_{0} r_{0}}\right)^{n-1-k} \frac{1}{1+2(k-n)}\left(\frac{u}{r_{0}}\right)^{k} \\
\approx & \sum_{n>0}\left(b_{n}+i a_{n}\right)\left(\frac{u}{r_{0}}\right)^{n-1} .
\end{aligned}
$$

The $N$ first terms of this equation can be written in matrix form

$$
\left(\begin{array}{c}
b_{1}+i a_{1} \\
\vdots \\
b_{N}+i a_{N}
\end{array}\right)=\left(\begin{array}{ccc}
A_{11} & \cdots & A_{1 N} \\
\vdots & \ddots & \vdots \\
0 & \cdots & A_{N N}
\end{array}\right)\left(\begin{array}{c}
\beta_{1}+i \alpha_{1} \\
\vdots \\
\beta_{N}+i \alpha_{N}
\end{array}\right) \text {, }
$$

with 


$$
\begin{aligned}
A_{m n} & =\frac{(n-1) !}{(m-1) !(n-m) ![1-2(m-n)]}\left(\frac{L_{S}{ }^{2}}{8 R_{0} r_{0}}\right)^{n-m}, \\
n & \geq m .
\end{aligned}
$$

The $a_{n}$ and $b_{n}$ can be measured with a stretched wire [29], as well as the magnetic length $L_{S}$ and on the radius of curvature $R_{0}$ which appear in the matrix $\mathbf{A}$. The pseudocurvilinear multipole coefficients $\beta_{n}$ and $\alpha_{n}$ can thus be obtained from the (pseudo)inverse $\mathbf{A}^{+}$of $\mathbf{A}$ :

$$
\left(\begin{array}{c}
\beta_{1}+i \alpha_{1} \\
\vdots \\
\beta_{N}+i \alpha_{N}
\end{array}\right)=\mathbf{A}^{+}\left(\begin{array}{c}
b_{1}+i a_{1} \\
\vdots \\
b_{N}+i a_{N}
\end{array}\right) .
$$

These results are valid in the case where the magnet is longitudinally centered on the wire, i.e., $\Delta y=0$. This is usually not true in practice: the term $y^{2}$ in Eq. (15) should be replaced by $(y-\Delta y)^{2}$. Inserting the longitudinal position in the equations lead to a more complex expression for the matrix coefficients:

$A_{m n}=\frac{(n-1) !\left[\left(\frac{L_{S}}{2}-\Delta y\right)^{2(n-m)+1}+\left(\frac{L_{S}}{2}+\Delta y\right)^{2(n-m)+1}\right]}{(m-1) !(n-m) ![1-2(m-n)] L_{S}\left(2 R_{0} r_{0}\right)^{n-m}}$,

$$
n \geq m \text {, }
$$

which simplifies to Eq. (18) if $\Delta y=0$.

One should note that the presence of binomial coefficients in the $A_{m n}$ makes the inversion of $\mathbf{A}$ unstable if $N$ is too large. For all $n>20$, we set $a_{n}$ and $b_{n}$ to zero to avoid inversion issues.

The coefficients obtained with Eq. (19) can easily be converted to pseudoelliptic multipole coefficients $\mathcal{E}_{k}$ using the matrix notations introduced in the Appendix:

$$
\left(\begin{array}{c}
\vdots \\
\mathcal{E}_{k} \\
\vdots
\end{array}\right)=\mathbf{M}_{E}{ }^{-1} \mathbf{M}_{C} \mathbf{A}^{+}\left(\begin{array}{c}
\vdots \\
b_{k}+i a_{k} \\
\vdots
\end{array}\right) \text {. }
$$

The methods described above were first tested with a 3D model of the DQ1 dipole quadrupole. Pseudomultipoles of the integrated field were given in Table II. Assuming $R_{0}=35.20 \mathrm{~m}$, enforcing $B L_{S 1}=0.5844 \mathrm{Tm}$ and using Eq. (5) to (14) lead to the values given in Table III. In Sec. II B, the quadrupole magnetic length, defined as the integrated gradient divided by the central gradient, was found equal to $1044 \mathrm{~mm}$, which is almost the same value as in Table III.

A $35.255 \mathrm{~m}$ bending radius was estimated from the integrals along straight lines. It is close to the nominal radius: the relative difference between the two radiuses is about $0.15 \%$. However, this requires further verification:
TABLE III. Determination of the magnet properties from integrals of the field along straight lines. The field integrals $I_{Z 01}, I_{Z 02}, I_{Z 11}, I_{Z 12}$ and the second field integrals $J_{Z 11}$ and $J_{Z 12}$ were computed with a $3 \mathrm{D}$ model of the DQ1 magnet, using the Radia software. The $J_{Z 110}$ and $J_{Z 120}$ integrals were computed using Eq. (6), the integrated gradient was obtained from Eq. (7), the quadrupole magnetic length was computed from Eq. (11) and the bending radius was determined using Eq. (14).

\begin{tabular}{lcc}
\hline \hline$I_{Z 01}$ & 0.63045 & $\mathrm{~T} \mathrm{~m}$ \\
$I_{Z 02}$ & 0.44049 & $\mathrm{~T} \mathrm{~m}$ \\
$I_{Z 11}$ & 0.53544 & $\mathrm{~T} \mathrm{~m}$ \\
$I_{Z 12}$ & 0.53544 & $\mathrm{~T} \mathrm{~m}$ \\
$J_{Z 11}$ & 0.41310 & $\mathrm{~T} \mathrm{~m}^{2}$ \\
$J_{Z 12}$ & 0.39009 & $\mathrm{~T} \mathrm{~m}^{2}$ \\
$J_{Z 110}$ & 0.41310 & $\mathrm{~T} \mathrm{~m}^{2}$ \\
$J_{Z 120}$ & 0.39010 & $\mathrm{~T} \mathrm{~m}$ \\
$G L_{S 2}$ & -37.991 & $\mathrm{~T}$ \\
$L_{S 2}$ & 1.0439 & $\mathrm{~m}$ \\
$R_{0}+\Delta R_{0}$ & 35.255 & $\mathrm{~m}$ \\
\hline \hline
\end{tabular}

any method giving a small $\Delta R_{0}$ would lead to a small difference between the nominal radius and the estimated one. To check this, the assumed value of $R_{0}$ in Eq. (5) to Eq. (14) was modified and the bending radius was estimated. The results plotted in Fig. 11 shows that the method remains accurate even if the assumed value of $R_{0}$ is wrong.

Table IV shows the pseudomultipole coefficients computed with Eq. (19) and allow a comparison to the pseudomultipoles integrated along curves parallel to the reference trajectory and to the pseudomultipoles integrated

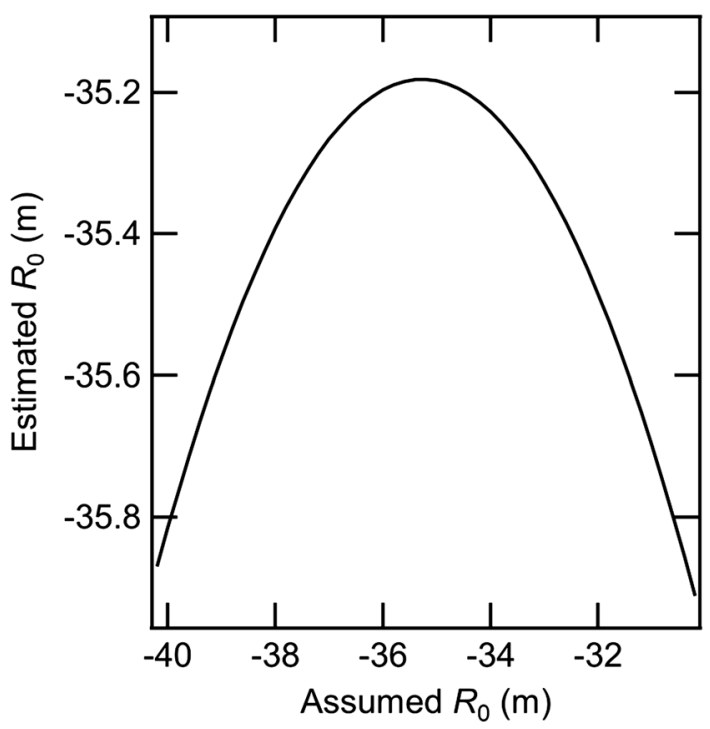

FIG. 11. Bending radius estimated from the field integral method versus assumed bending radius. If the assumed bending radius is wrong by $5 \mathrm{~m}$, the error in the bending radius estimation is about $0.7 \mathrm{~m}$. A $1 \mathrm{~m}$ error in the assumed bending radius gives a $3 \mathrm{~cm}$ error in the estimation (from 3D simulations with Radia). 
TABLE IV. Simulated pseudomultipoles of the integrated field computed with different methods. (Trajectory): The field was integrated along curves parallel to the reference trajectory. (Parabola): The field was integrated along parabolas with $35.255 \mathrm{~m}$ curvature radius. (Straight to parabola): The field was integrated along straight lines, then Eq. (19) was used in order to compute pseudomultipoles integrated along a parabola. The horizontal and the vertical field components were sampled on circles centered on the parabola and with symmetry axis parallel to the magnet axis.

\begin{tabular}{lccc}
\hline \hline & Trajectory & Parabola & Straight to parabola \\
\hline $\begin{array}{l}\text { Normalization } \\
\text { field (T mm) }\end{array}$ & 584.38 & 585.72 & 585.72 \\
$n$ & $\beta_{n}$ & $\beta_{n}$ & $\beta_{n}$ \\
1 & 10000 & 10000 & 10000 \\
2 & -4550.8 & -4548.0 & -4548.5 \\
3 & 2.5 & 2.1 & 4.1 \\
4 & 3.7 & 3.8 & 3.7 \\
5 & -2.7 & -2.6 & -2.9 \\
6 & -9.0 & -9.3 & -9.6 \\
7 & 2.9 & 2.8 & 1.9 \\
8 & 9.5 & 10.1 & 11.0 \\
\hline \hline
\end{tabular}

along parabolas. The agreement between the different methods is $2 \times 10^{-4}$ or better for all coefficients.

The reference trajectory is well approximated by a parabola in the interior of the magnet but this is not the case in the fringe field region. However, Table IV demonstrates that integrals of the field along parabolas are good approximations of the field integrals along the reference trajectories.

\section{B. Magnetic measurement benches}

A Hall probe bench designed at the ESRF for undulator measurements [33] was used for measuring locally the magnetic field. The Hall sensors were calibrated versus a nuclear magnetic resonance probe in a dipole magnet.

Then, the dipole-quadrupole magnets were measured with stretched wire benches. These benches are made with Newport ILS-100CC and IMS-100V linear stages, driven by a Newport XPS motion controller. The stages were calibrated with an interferometer and their accuracy is in the range of $1 \mu \mathrm{m}$. The voltage induced on the wire was measured with a Keitley 2182 voltmeter. A $0.1 \mathrm{~mm}$ diameter Ti-6Al-4V wire was used. Please refer to Ref. [34] for a more detailed presentation of the ESRF stretched wire benches.

The following measurement sequence was implemented on the measurement bench: (i) measurement of the field integrals and the second field integrals $I_{Z 01}, I_{Z 02}, I_{Z 11}, I_{Z 12}$, $I_{X 11}, I_{X 12}, J_{Z 11}, J_{Z 12}, J_{X 11}$ and $J_{X 12}$ and determination of the gradient, longitudinal position, yaw and pitch angle, magnetic length and curvature, (ii) measurement of the integrated field at 128 points on a circle with $9 \mathrm{~mm}$ diameter, i.e., at the maximum radius compatible with the geometry of the curved poles. All the measurement sequences and the analysis routines are part of the open source software SW Lab currently developed at the ESRF [35].

\section{Results}

Two kinds of measurements were performed on the DQ magnets: local measurements with a Hall probe bench, and integral measurements with a stretched wire bench.

The field and gradient are shown in Fig. 12. These measurements were used to determine the dipole length $L_{1 \text { MEAS }}=1056 \mathrm{~mm}$ (simulated value $1054.7 \mathrm{~mm}$ ) and the quadrupole length $L_{2 \text { MEAS }}=1045 \mathrm{~mm}$ (simulated value $1044.0 \mathrm{~mm}$ ). The magnetic lengths were defined as the integral of the field divided by its central value.

The bending radius of the poles was also determined from the local measurements, assuming that the vertical field $B_{Z}$ is homogeneous on the trajectory and excluding the extremities of the magnet. The best fitted value of the curvature radius is $35.06 \mathrm{~m}$, which is close to the $35.20 \mathrm{~m}$ specified mechanical radius. Considering the deflection angle of the DQ1, the error on the sagitta of the magnet is (35.20-35.06) $(1-\cos 0.0292 / 2) \approx 15 \mu \mathrm{m}$, which is well
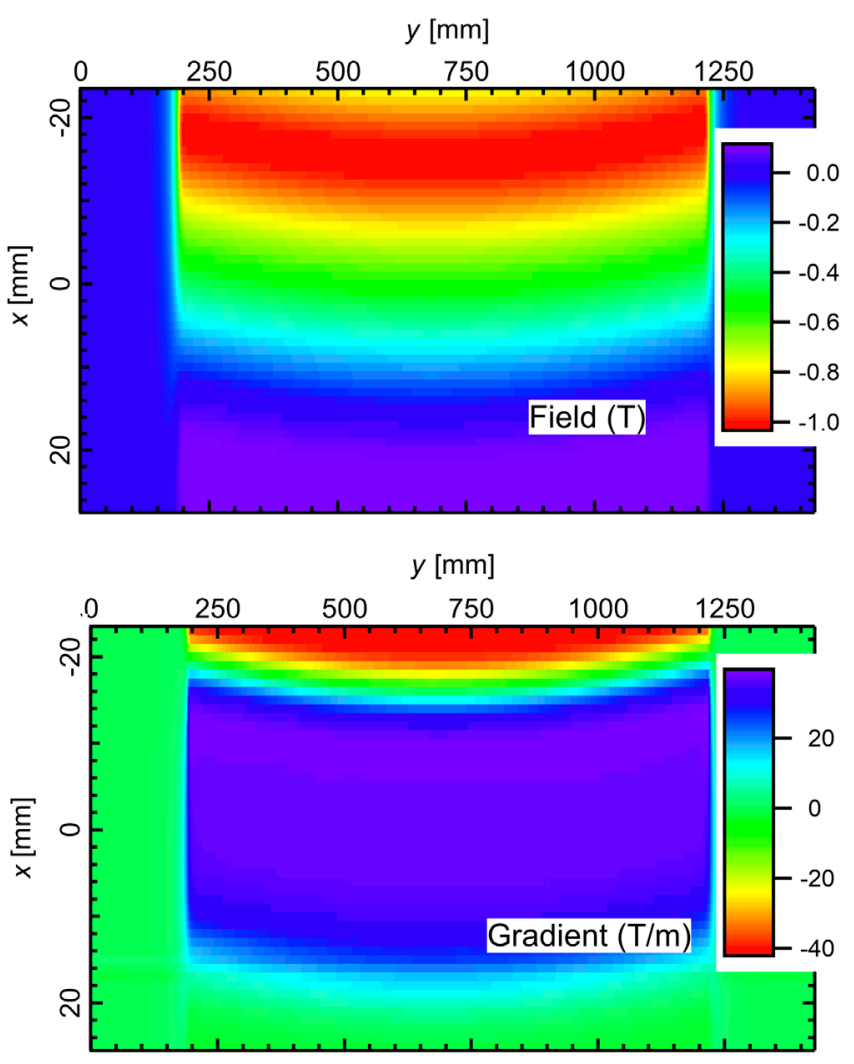

FIG. 12. Top: Magnetic field of the DQ1 preseries magnet DQ1-002, measured with a Hall probe bench. Step size: $1 \mathrm{~mm}$. Bottom: Field gradient obtained by differentiating the magnetic field. 
within the tolerances. It should be noted that vertical field along the reference arc is not perfectly flat, as shown in Fig. 7. Thus, the "magnetic" bending radius determined from the field maps is not equal to the mechanical bending radius of the poles.

Due to the limited accuracy of our Hall probe bench and to the noise introduced by the differentiation of the field, we were not able to determine the gradient homogeneity with this method.

The dipole-quadrupole magnets were then measured with a stretched wire bench using the method described in Sec. III. 1. The results presented below are for one particular magnet, the DQ1-063, which was measured in details.

The magnet current was adjusted first, in order to reach the integrated gradient specification. At least two iterations are needed to set the gradient to its nominal value. The determination of the current must be done before the alignment and fiducialization because the field at a given position strongly depends on this current. In all magnets, the current corresponding to the nominal gradient was found lower than its simulated value by about $3 \%$.

The measurement sequence was repeated 16 times with the same initial conditions in order to determine the accuracy of the alignment. The standard deviation for the magnet center was $1 \mu \mathrm{m}$ for the horizontal offset, $4 \mu \mathrm{m}$ for the vertical offset, and $0.16 \mathrm{~mm}$ for the longitudinal offset. The standard deviation is larger for the vertical offset due to the thermal drift of the magnet. The nominal diameter of the wire is $100 \pm 5 \mu \mathrm{m}$, which brings additional uncertainties. Its sagitta was estimated to $13 \mu \mathrm{m}$ and was corrected at the end of the measurement. But the main contributors to the alignment uncertainties are the laser trackers used for transferring the positions of the wire holders to the alignment references of the magnets. The rms error of the coordinate measurements was found to be $18 \mu \mathrm{m}$, using two laser trackers installed at two different positions [36].

The standard deviation for the pitch and yaw angles was about $0.06 \mathrm{mrad}$. It should be mentioned that the repeatability of the pitch and yaw angle measurements strongly depends on the measurement setup. In particular, measuring a short magnet with a long wire would lead to poor accuracy. A $1.64 \mathrm{~m}$ long wire was used for the experiment described here.

The quadrupole length of this magnet was estimated to $L_{S}=1047.7 \pm 1.4 \mathrm{~mm}$ and the radius of curvature of its poles was estimated to $R_{0} \approx 35.12 \pm 0.08 \mathrm{~m}$. This radius is pretty close to the 35.21 nominal radius and the difference between the measured and the nominal sagitta is only $0.01 \mathrm{~mm}$. This value is also in good agreement with the radius measured on the preseries with local field measurements.

The multipoles coefficients of the integral of the field along a parabola are given in Table V. The large difference between $b_{2}$ (integral along a straight line) and $\beta_{2}$ (integral along a parabola) is the result of the transverse offset $\Delta x \approx \mathcal{S} / 3$ expected from Eq. (9). The measured value of $\beta_{2}$ is slightly different from the simulated value given in Table II. It comes from a change of the EBS lattice: the gradient of the dipole quadrupole in the final lattice is $0.9 \%$ higher than the gradient used to design the magnet while the field was kept constant. The new working point was obtained by changing the current and by changing the transverse offset of the magnet by $0.13 \mathrm{~mm}$. The first multipole coefficients are in agreement with the nominal values and tolerances, but the $\beta_{5}$ and $\beta_{6}$ differ significantly from the simulations. Further magnetic simulations have shown that the measured value of $\beta_{6}$ cannot be explained by a deformation of the magnet resulting in a closure of its gap. A possible explanation is sketched in Fig. 13. Due to the curvature of the magnet, a small part of the GFR is out of the measured volume at the extremities of the magnets. Measuring the magnet with a different transverse offset, e.g., $\Delta X=\mathcal{S} / 3$, would imply to reduce the measurement radius and would not necessarily solve this issue. Another possibility, to be further developed, would be to combine measurements at two different offsets and radii. Combining several measurements is feasible using the matrix formalism developed in [29].

TABLE V. Measured multipole coefficients of the field integrated along a straight line $\left(a_{n}, b_{n}\right)$ expressed at $7 \mathrm{~mm}$, and curvilinear multipoles $\left(\beta_{n}, \alpha_{n}\right)$ at the same radius. All multipoles were normalized to the normal dipole. All measurements were done on the DQ1-063 magnet. The errors are standard deviations estimated from repeated measurements.

\begin{tabular}{lcccc}
\hline \hline$n$ & $b_{n}$ & $\beta_{n}$ & $a_{n}$ & $\alpha_{n}$ \\
\hline Normalization field (T mm) & 534.68 & 584.20 & 534.67 & 584.20 \\
1 & 10000 & 10000 & $7.0 \pm 0.7$ & $6.4 \pm 0.7$ \\
2 & $5020.8 \pm 1.0$ & $4590.9 \pm 0.9$ & $0.4 \pm 0.3$ & $-0.1 \pm 0.3$ \\
3 & $-3.0 \pm 0.2$ & $-5.0 \pm 0.2$ & $1.3 \pm 0.2$ & $1.8 \pm 0.2$ \\
4 & $-4.1 \pm 0.3$ & $-5.6 \pm 0.4$ & $-1.1 \pm 0.1$ & $-1.3 \pm 0.2$ \\
5 & $-0.7 \pm 0.3$ & $5.0 \pm 0.2$ & $0.4 \pm 0.2$ & $0.4 \pm 0.2$ \\
6 & $4.7 \pm 0.1$ & $19.1 \pm 0.4$ & $-0.1 \pm 0.1$ & $0.4 \pm 0.1$ \\
7 & $13.95 \pm 0.3$ & $8.3 \pm 0.5$ & $-0.5 \pm 0.1$ & $-0.6 \pm 0.1$ \\
8 & $-3.1 \pm 0.1$ & $-9.1 \pm 0.2$ & $0.2 \pm 0.1$ & $0.3 \pm 0.2$ \\
\hline \hline
\end{tabular}




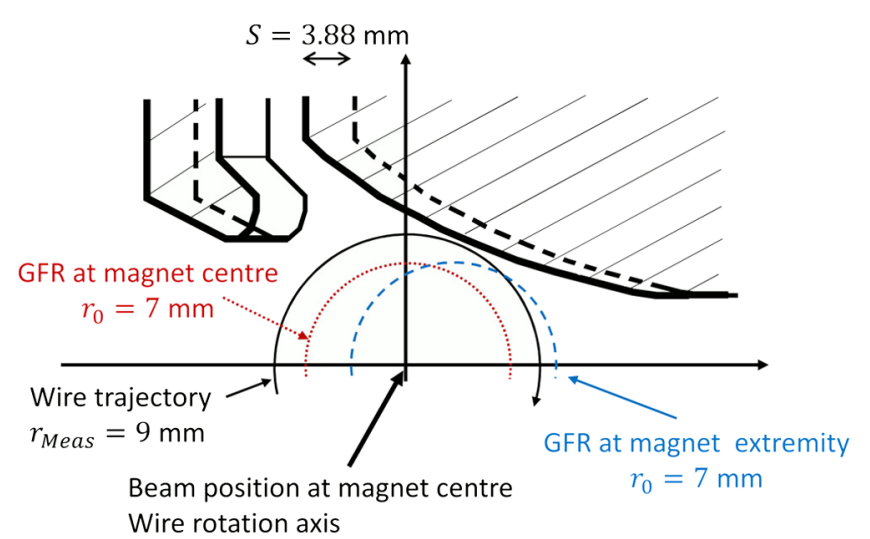

FIG. 13. Cut view of a DQ1 from its center, showing the trajectory of the wire and the GFR in the middle and at the extremities. The wire rotation axis was tangent to the reference trajectory in the middle of the magnet. At the magnet extremities, a part of the GFR is out of the measurement radius. This may be a cause of the discrepancies between simulations and measurements for the higher order harmonics.

The integrated gradient and its homogeneity can be computed from the multipoles. Homogeneity contours are shown in Fig. 14. The normalized gradient error $\Delta G / G$ is well below the $1 \%$ specification. It is under $0.1 \%$ within a $\pm 5.3 \mathrm{~mm}$ range in the horizontal direction. (Magnetic simulation gave a $0.1 \%$ homogeneity within a $\pm 6.1 \mathrm{~mm}$ range, see Fig. 5.)

\section{EFFECT OF THE MAGNET'S CURVATURE}

In the above discussion, the field of the magnet was approximated by a set of slices. The field in each slice was assumed to be an analytic function. For straight magnets this approximation gives the correct integrals of the field, even if it hides the fringe field effect. (We discussed some effects of the fringe field of quadrupole magnets in Ref. [4].)

In this discussion, we will focus on the integral of the fields along the reference trajectory of the electrons [Fig. 4(b)]. The field integral along a curved path differs from an analytic function. Stated differently, reconstructing the field from Fourier coefficients estimated on the boundary of a given region would lead to errors inside this region. For a given magnet model, these errors can be simulated easily. Complex field integrals $I_{k}=\int\left(B_{z}+\right.$ $\left.i B_{x}\right) d s$ were computed at $u_{k}=x_{k}+i z_{k}=\rho_{0} \operatorname{Exp}\left(i \theta_{k}\right)$, where $\rho_{0}$ is the radius of the good field region, $\theta_{k}=$ $2 \pi k / K$ and $0 \leq k<K$. The path length depends on $u_{k}$ and the reference trajectory corresponds to $u=0$. In the case these field integrals would be an analytic function of $u$, the series coefficients would be obtained from the Fourier transform of the signal $\mathbf{I}=\left(I_{1}, \ldots, I_{K}\right)$, as is usually done for multipole analysis. Let us denote $\hat{a}_{n}$ and $\hat{b}_{n}$ the series coefficients obtained from the Fourier transform of $\mathbf{I}$. Then, a field integral error can be estimated at each point $(x, z)$ inside the good field region:

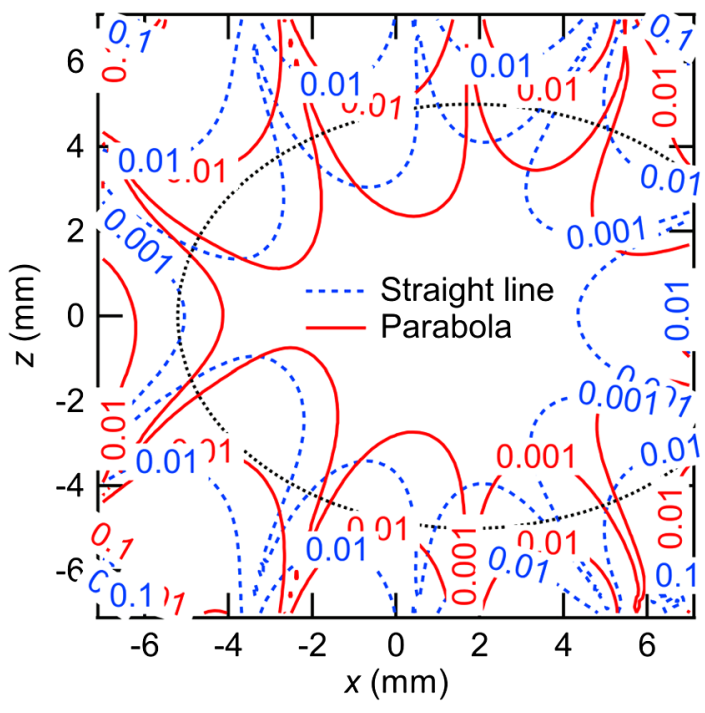

FIG. 14. Homogeneity contours of the magnetic field gradient integrated along a straight line and along a parabola whose minimal radius of curvature is equal to the measured bending radius of the magnet. The dotted line in black indicates the $14 \mathrm{~mm}$ x $10 \mathrm{~mm}$ good field region. In the horizontal plane, the gradient integrated along the parabola has a homogeneity better than $0.1 \%$ in a $\pm 5.3 \mathrm{~mm}$ range.

$$
\varepsilon(x, z)=\frac{\left|I(x, z)-\sum_{n \geq 1}\left(\hat{b}_{n}+i \hat{a}_{n}\right)\left(\frac{x+i z}{\rho_{0}}\right)^{n-1}\right|}{\max |I(x, z)|} .
$$

This error is shown in Fig. 15. It demonstrates that within the good field region the integral of the field along paths

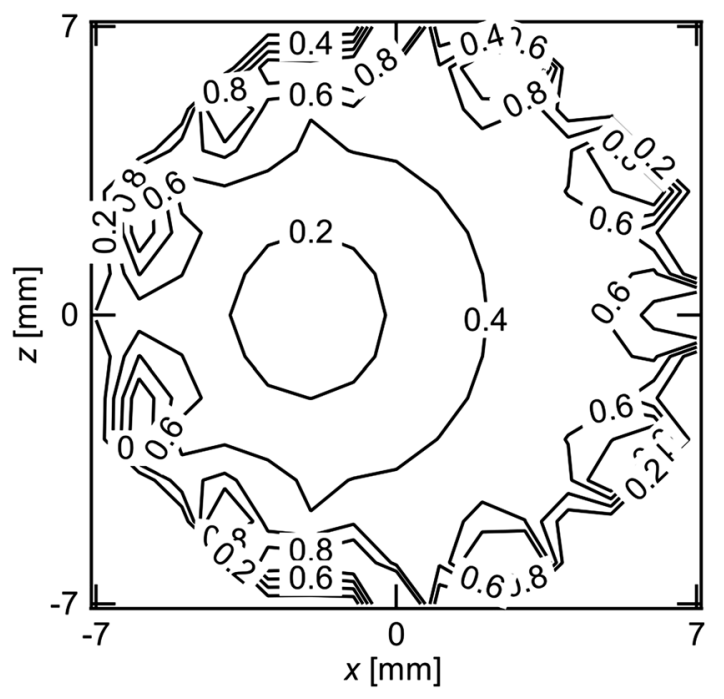

FIG. 15. Contour plot of the error $10^{4} \varepsilon(x, z)$ defined in Eq. (22). This error is the normalized difference between the integral of the field along a path parallel to the reference trajectory, and an analytic function whose series coefficients were evaluated from the values of the integrated field on a $7 \mathrm{~mm}$ radius circle. The higher values of the error, on the boundary, are due to the truncation of the series to its ten first terms. 
parallel to the trajectory can be approximated with a relative error below $10^{-4}$ with a Fourier expansion.

Solutions of the Laplace equation in curvilinear coordinates, i.e., curvilinear multipoles, have been studied by others. Cylindrical multipoles were first proposed by McMillan [37], rediscovered independently by Mane [38] and studied in more details by Zolkin [39]. Toroidal field multipoles were studied by other authors. Schnizer et al. introduced a local toroidal coordinate system and shown the curvature results in distortions of the standard field multipoles [24,40]. Wolski and Herrod have recently published an expression of the potential in toroidal coordinates [41]. We implemented a similar toroidal multipole expansion and we computed the error between the field integral and a sum of toroidal multipoles. For the magnet we investigated, the relative error was again in the $10^{-4}$ range. As the toroidal multipoles are a solution of the Laplace equations in this geometry, the field at a given point should be equal to the toroidal multipole series at the same point. This indicates that the accuracy of the computations is about $10^{-4}$.

\section{CONCLUSION}

Off-axis quadrupoles are suitable to achieve the specifications of the combined dipole quadrupoles to be integrated in the new generation of storage ring based light sources. These magnets can be asymmetric in order to reduce their electrical power consumption. Such magnets were designed and constructed for the EBS. The shapes of the magnets were optimized in order to obtain a good gradient homogeneity within an elliptic good field region. The magnets are single sided and produce almost no field on their outer side. Correction coils were installed in order to tune the field and the gradient independently.

Stretched wire magnetic measurement methods were developed for the magnet alignment and for measuring its main parameters, i.e., magnetic length, gradient, pole bending radius and pseudomultipole coefficients. The accuracy of these methods was demonstrated with 3D simulations. The parameters measured on one of the dipolequadrupole magnet series were presented. The alignment accuracy was very good, the fiducialization errors being dominated by laser tracker uncertainties. The multipole coefficients were in good agreement with the simulation results and tolerances up to the octupole, but the decapole and dodecapole are significantly higher than their simulated value. These discrepancies may be explained by the imperfect matching of the volume measured by the wire and the GFR volume. Combining two or more measurements performed at different offsets and radii would allow overcoming this issue but would need further developments. It should be noted that the stretched wire methods developed in the paper may be adapted to rotating coil measurements.
If the field integral is evaluated on a curved path rather than on a straight line, it is not an analytic function. However, we have shown that given the large radius of curvature and the small radius of the good field region for the magnets described here, the relative error between the integral of the field and its analytic approximation is not larger than $10^{-4}$.

\section{ACKNOWLEDGMENTS}

The authors would like to thank Dr. C. Benabderrahmane who followed up the production of the DQ series magnets, and the magnet company Tesla Engineering Ltd., UK, who produced all of the dipole quadrupoles for the EBS.

\section{APPENDIX}

\section{Standard multipoles and elliptic multipoles for straight magnets}

The field of multipole accelerator magnets is often expressed as a multipole series: the magnetic field is described with a small number of coefficients, making its representation easier. The most common multipoles are the 2D "straight" multipoles obtained by integration of the field along a line.

Within the aperture of a multipole magnet, the Maxwell equations reduce to $\nabla \cdot \mathbf{B}=0$ and $\nabla \times \mathbf{B}=0$. The integral of a magnetic field along a straight line is a $2 \mathrm{D}$ field, for which the Maxwell equations are equivalent to the CauchyRiemann equations. The complex field $B=B_{Z}+i B_{X}$, where $B_{Z}$ and $B_{X}$ are the vertical and horizontal components of the integral of the field along a straight line, can be expressed as the Taylor series

$$
B=\sum_{n=1}^{\infty}\left(b_{n}+i a_{n}\right)\left(\frac{u}{\rho_{0}}\right)^{n-1},
$$

where $u=x+i z$ is the complex function, $b_{n}$ and $a_{n}$ are the normal and skew 2n-pole coefficients and $\rho_{0}$ is the reference radius.

The apertures of dipole magnets are often noncircular. Elliptic multipoles have been introduced by Schnizer et al. $[23,24]$ for describing the dipole fields on noncircular aperture. The elliptic coordinates are defined as $x=$ $e \cosh \eta \cos \psi$ and $z=e \sinh \eta \sin \psi$, where $e$ is the eccentricity, $0 \leq \eta<\infty$ and $-\pi \leq \psi<\pi$. Cartesian coordinates are transformed to elliptic coordinates by the conformal map

$$
w=\eta+i \psi=\operatorname{Arccosh}(u / e) .
$$

Inserting this in standard multipole series and using Moivre's formula and the binomial theorem, then rearranging the terms and introducing new notations leads to 


$$
B=\frac{E_{0}}{2}+\sum_{n=1}^{\infty} E_{n} \frac{\cosh (n w)}{\cosh \left(n \eta_{0}\right)}
$$

The $E_{n}$ 's are the elliptic multipole coefficients while $\eta_{0}$ is a normalization factor.

If the summation in Eq. (A1) is truncated to its first terms, the field at a given set of points can be expressed in matrix form:

$$
\mathbf{B}=\mathbf{M}_{\mathbf{E}} \mathbf{E},
$$

where $B_{i}=B\left(w_{i}\right)$, the $E_{j}$ 's are the complex elliptic multipole coefficients, $1 \leq i \leq M, 1 \leq j \leq N$ and

$$
M_{E i j}= \begin{cases}\frac{1}{2} & \text { for } j=1 \\ \mathscr{B} \frac{\cosh \left[(j-1) w_{i}\right]}{\cosh \left[(j-1) \eta_{0}\right]} & \text { for } j>1 .\end{cases}
$$

This multipole expansion has been used for optimizing the field on an elliptical good field region.

Similarly, the field in polar coordinates writes $\mathbf{B}=$ $\mathbf{M}_{\mathbf{C}} \mathbf{C}$, where the $C_{j}$ 's are the complex multipole coefficients and $M_{C i j}=\left(u_{i} / \rho_{0}\right)^{j-1}$. One can choose the points $i$ such that the matrix $\mathbf{M}_{\mathbf{C}}$ is invertible. In such a case, the elliptic multipoles transform to the circular ones according to

$$
\mathbf{C}=\mathbf{M}_{\mathbf{C}}{ }^{-1} \mathbf{M}_{\mathbf{E}} \mathbf{E} .
$$

This yields $\|\mathbf{C}\|_{F} \leq\left\|\mathbf{M}_{\mathbf{C}}{ }^{-1} \mathbf{M}_{\mathbf{E}}\right\|_{F}\|\mathbf{E}\|_{F}$ where $\|\mathbf{A}\|_{F}$ is the Frobenius norm of $\mathbf{A}$. The norm $K=\left\|\mathbf{M}_{\mathbf{C}}{ }^{-1} \mathbf{M}_{\mathbf{E}}\right\|_{F}$ depends only on the positions at which the field is computed, i.e., on the good field region, so the minimization of the elliptic multipole errors $\left\|\boldsymbol{\varepsilon}_{E}\right\|=\left\|\mathbf{E}-\mathbf{E}_{\text {TARGET }}\right\|$ for a given good field region yields to small circular multipole errors: $\left\|\boldsymbol{\varepsilon}_{C}\right\| \leq K\left\|\boldsymbol{\varepsilon}_{E}\right\|$.

[1] L. Farvacque, N. Carmignani, J. Chavanne, A. Franchi, G. Le Bec, S. Liuzzo, B. Nash, T. Perron, P. Raimondi, A lowemittance lattice for the ESRF, in Proceedings of the 4th International Particle Accelerator Conference, IPAC2013, Shanghai, China, 2013 (JACoW, Shanghai, China, 2013), pp. 79-81.

[2] J. C. Biasci, J. F. Bouteille, N. Carmignani, J. Chavanne, D. Coulon, Y. Dabin, F. Ewald, L. Farvacque, L. Goirand, M. Hahn et al., A low-emittance lattice for the ESRF, Synchrotron Radiat. News 27, 8 (2014).

[3] G. Le Bec, J. Chavanne, F. Villar, C. Benabderrahmane, S. Liuzzo, J.-F. Bouteille, L. Goirand, L. Farvacque, J.-C. Biasci, and P. Raimondi, Magnets for the ESRF diffraction limited light source project, IEEE Trans. Appl. Supercond. 26, 1 (2016).

[4] G. Le Bec, J. Chavanne, C. Benabderrahmane, L. Farvacque, L. Goirand, S. Liuzzo, P. Raimondi, and F. Villar, High gradient quadrupoles for low emittance storage rings, Phys. Rev. Accel. Beams 19, 052401 (2016).
[5] J. Chavanne and G. Le Bec, Prospects for the use of permanent magnets in future accelerator facilities, in Proceeding of the International Particle Accelerator Conference 14 (IPAC14), Dresden, Germany (JACoW, Dresden, Germany, 2014), pp. 968-973.

[6] E. D. Courant, M. S. Livingston, and H. S. Snyder, The strong-focusing synchrotron-A new high energy accelerator, Phys. Rev. 88, 1190 (1952).

[7] Y. Chen, D. E. Kim, W. Kang, F. S. Chen, M. Yang, Z. Zhang, B. G. Yin, and J.X. Zhou, Designs and measurements of gradient dipole magnets for the upgrade of Pohang Light Source, Nucl. Instrum. Methods Phys. Res., Sect. A 682, 85 (2012).

[8] D. Einfeld, M. Melgoune, G. Beneditti, M. De Lima, J. Marcos, M. Munoz, M. Pont, Modelling of gradient bending magnets for the beam dynamics studies at ALBA, in Proceedings of the 22nd Particle Accelerator Conference, PAC-2007, Albuquerque, NM (IEEE, New York, 2007), pp. 1076-1078.

[9] L. Dallin, I. Blomqvist, D. Lowe, J. Swirksy, J. Campmany, F. Goldie, J. Coughlin, Gradient dipole magnets for the Canadian Light Source, in Proceedings of the 8th European Particle Accelerator Conference, Paris, 2002 (EPS-IGA and CERN, Geneva, 2002).

[10] J. A. Eriksson, A. Andersson, M. Johansson, D. Kumbaro, S. C. Leemann, C. Lenngren, P. Lilja, F. Lindau, L.-J. Lindgren, L. Malgren et al., The MAX IV synchrotron light source, in Proceedings of the 2nd International Particle Accelerator Conference, San Sebastian, Spain (EPS-AG, Spain, 2011), pp. 3026-3028.

[11] M. Johansson, B. Anderberg, and L. J. Lindgren, Magnet design for a low-emittance storage ring, J. Synchrotron Radiat. 21, 884 (2014).

[12] M. Yoon, J. Corbett, M. Cornacchia, J. Tanabe, and A. Terebilo, Analysis of a storage ring combinedfunction magnet: Trajectory calculation and alignment procedure, Nucl. Instrum. Methods Phys. Res., Sect. A 523, 9 (2004).

[13] M. Sjöström, E. Wallén, M. Eriksson, and L. J. Lindgren, The MAX III storage ring, Nucl. Instrum. Methods Phys. Res., Sect. A 601, 229 (2009).

[14] M. Borland, V. Sajaev, Y. Sun, and A. Xiao, Hybrid sevenbend-achromat lattice for the advanced photon source upgrade, in Proceeding of the International Particle Accelerator Conference 15 (IPAC15), Richmond, USA, 2015, pp. 1776-1779.

[15] C. Steier, J. Byrd, H. Nishimura, D. Robin, S. De Santis, F. Sannibale, C. Sun, M. Venturini, W. Wan, Physics design progress towards a diffraction limited upgrade of the ALS, in Proceeding of the International Particle Accelerator Conference 16 (IPAC16), Busan, Korea, 2016, pp. 29562958.

[16] H. Tanaka, T. Ishikawa, S. Goto, S. Takano, T. Watanabe, and M. Yabashi, SPring-8 upgrade project, in Proceeding of the International Particle Accelerator Conference 16 (IPAC16), Busan, Korea, 2016, pp. 2867-2870.

[17] E. Karantzoulis, The diffraction limited light source ELETTRA 2.0, in Proceeding of the International Particle Accelerator Conference 17 (IPAC17), Copenhagen, Denmark, 2017, pp. 2660-2662. 
[18] B. Parker, N. L. Smirnov, and L. M. Tkachenko, Development of septum quadrupole prototype for DESY upgrading luminosity, Nucl. Instrum. Methods Phys. Res., Sect. A 434, 297 (1999).

[19] P. Elleaume, O. Chubar, and J. Chavanne, Computing 3D magnetic fields from insertion devices, in Proceedings of the Particle Accelerator Conference, Vancouver, BC, Canada, 1997 (IEEE, New York, 1997), pp. 3509-3511.

[20] O. Chubar and P. Elleaume, Accurate and efficient computation of synchrotron radiation in the near field region, in Proceedings of the 6th European Particle Accelerator Conference, Stockholm, 1998 (IOP, London, 1998).

[21] G. Le Bec, J. Chavanne, and P. N'gotta, Shape optimization for the ESRF II magnets, in Proceeding of the International Particle Accelerator Conference 14, IPAC14, Dresden, 2014, pp. 1232-1234.

[22] S. Russenschuck, Field Computation for Accelerator Magnets (Wiley-VCH, New York, 2010).

[23] P. Schnizer, B. Schnizer, P. Akishin, and E. Fischer, Theory and application of plane elliptic multipoles for static magnetic fields, Nucl. Instrum. Methods Phys. Res., Sect. A 607, 505 (2009).

[24] P. Schnizer, B. Schnizer, P. Akishin, and E. Fischer, Plane elliptic or toroidal multipole expansions for static fields. Applications within the gap of straight and curved accelerator magnets, COMPEL 28, 1044 (2009).

[25] L. Walckiers, The harmonic-coil method, in CERN Accelerator School: Magnetic measurement and alignment, Montreux, Switzerland, 1992, pp. 138-166.

[26] J. DiMarco and J. Krzywinski, MTF single stretched wire system, Fermilab MTF-96-0001, 1996.

[27] J. DiMarco, H. Glass, M. J. Lamm, P. Schlabach, C. Sylvester, J. C. Tompkins, and I. Krzywinski, Field alignment of quadrupole magnets for the LHC interaction regions, IEEE Trans. Appl. Supercond. 10, 127 (2000).

[28] D. Zangrando and R. P. Walker, A stretched wire system for accurate integrated magnetic field measurements in insertion devices, Nucl. Instrum. Methods Phys. Res., Sect. A 376, 275 (1996).

[29] G. Le Bec, J. Chavanne, and C. Penel, Stretched wire measurement of multipole accelerator magnets, Phys. Rev. ST Accel. Beams 15, 022401 (2012).

[30] G. Deferme, Advances in stretched wire systems at CERN, in IMMW19, Hsinchu, Taiwan, 2015.

[31] J. DiMarco, Recent PCB rotating coil developments at Fermilab, in IMMW19, Hsinchu, Taiwan, 2015.

[32] A. K. Jain, Magnet alignment challenges for an MBA storage ring, in DLSR, Lemont, USA, 2014.

[33] J. Chavanne and P. Elleaume, Technology of insertion devices, in Undulators, Wigglers and their Applications, edited by H. Onuki and P. Elleaume (Taylor \& Francis, London, 2003), pp. 148-213.

[34] G. Le Bec, Overview of magnetic measurement activities at the ESRF, in IMMW20, Didcot, UK, 2017.

[35] SW Lab, https://gitlab.esrf.fr.

[36] D. Martin, Alignment of the ESRF Extremely Brilliant Source (EBS), in IWAA, Chicago, USA, 2018.

[37] E. M. McMillan, Multipoles in cylindrical coordinates, Nucl. Instrum. Methods 127, 471 (1975).

[38] S. R. Mane, Solutions of Laplace's equation in two dimensions with a curved longitudinal axis, Nucl. Instrum. Methods Phys. Res., Sect. A 321, 365 (1992).

[39] T. Zolkin, Sector magnets or transverse electromagnetic fields in cylindrical coordinates, Phys. Rev. Accel. Beams 20, 043501 (2017).

[40] P. Schnizer, E. Fischer, and B. Schnizer, Cylindrical circular and elliptical, toroidal circular and elliptical multipoles fields, potentials and their measurement for accelerator magnets, arXiv:1410.8090.

[41] A. Wolski and A. T. Herrod, Explicit symplectic integrator for particle tracking in s-dependent static electric and magnetic fields with curved reference trajectory, Phys. Rev. Accel. Beams 21, 084001 (2018). 